\title{
Laryngeal Neurofibroma
}

\section{Anatoli P*, Melina F and Petros S \\ Department of ENT, Hygeia Hospital, Greece}

*Corresponding author: Pataridou Anatoli, Department of ENT, Hygeia Hospital, Greece, Tel: 6932291793; Email: anatolipataridou@windowslive.com

\section{Case Report \\ Volume 4 Issue 1}

Received Date: June 19, 2019

Published Date: June 28, 2019

DOI: $10.23880 /$ ooaj-16000181

\section{Abstract}

Introduction: Neurofibromatosis is a genetic disease characterized by tumours of the nervous system (usually benign ones) and is classified in 3 categories: neurofibromatosis type 1 (NF1) known also as Von Recklinghausen Disease, neurofibromatosis type 2 (NF2) and swanommatosis. NF type 1 is the most common type of neurofibromatosis and is related with the presence of laryngeal neurofibromas.

Objective: Presentation of a rare clinical report of laryngeal neurofibroma and its non-invasive treatment.

Methods \& Results: Patient of 24 years with dyspnea, hoarseness of the voice and difficulty in swallowing with medical history of NF1. Surgical excision of the tumor with the use of $\mathrm{CO}_{2}$ laser, without external approach and with mild and easy postoperative time.

Conclusion: Laryngeal neurofibroma is a rare benign tumor with prevalence 3:2 in girls and with common finding in the supraglottic, arytenoid and aryteoepiglottic folds, all areas full of neural fibers. It can be easily resected having excellent results with the use of $\mathrm{CO}_{2}$ laser.

Keywords: Neurofibromatosis; Laryngeal neurofibroma; Fibrolaryngoscopy; Tumours

Abbreviations: CAGR: Compound Annual Growth Rate.

\section{Introduction}

Neurofibromatosis is a genetic disease characterized by tumours of the nervous system. The tumours can be developed in every point of the nervous system, on the brain and the spinal spine. Usually they consist of benign tumours but that they can degenerate into malign ones. Neurofibromatosis is classified in 3 categories: neurofibromatosis type 1 (NF1) known also as von Recklinghausen disease, type 2 (NF2) and swanommatosis. NF type 1 is the most common type of neurofibromatosis and is related with the presence of laryngeal neurofibromas. NF-1, is an autosomal dominant disease with an incidence of 1 in 2,600 to 3,000 individuals [1]. Laryngeal neurofibromas are extremely rare, presented in 0.03 to $0.1 \%$ of benign tumours of the larynx [2].

In this case report we present a 24-year old man with NF-1 who presented with dyspnea, hoarseness of the voice, difficulty in swallowing and will discuss the surgical treatment. The endoscopic examination revealed a supraglottic mass. 


\section{Otolaryngology Open Access Journal}

\section{Case Report}

The 24 year old patient with known medical history of NF1, was presented with hoarseness of the voice, difficulty in breathing and swallowing. Neck examination did not reveal any neck swelling or palpable mass. He went through a fibrolaryngoscopy that revealed a supraglottic mass occupying the right true vocal cord and partially occluding the laryngeal lumen (Figure 1).

The patient has also presented in the past a glioma of the optic nerve for which he was treated with chemotherapy, plexiform neurofibromas of the neck, multiple neurofibromas of the supclavicolar region, intercostal spaces, of the scrotum, the spinal spine and an operated suboccipital neurofibroma.

A CT scan of the neck revealed a well-circumscribed mass and showed the exact position and extension of it $(2.4 \mathrm{~cm}$ anteroposterior diameter, $1.27 \mathrm{~cm}$ laterolateral diameter) (Figures 2A \& 2B). Routine laboratory tests, including complete blood count, erythrocyte sedimentation rate, and bleeding profile were normal. There was no clinical evidence of inflammatory and autoimmune disease.

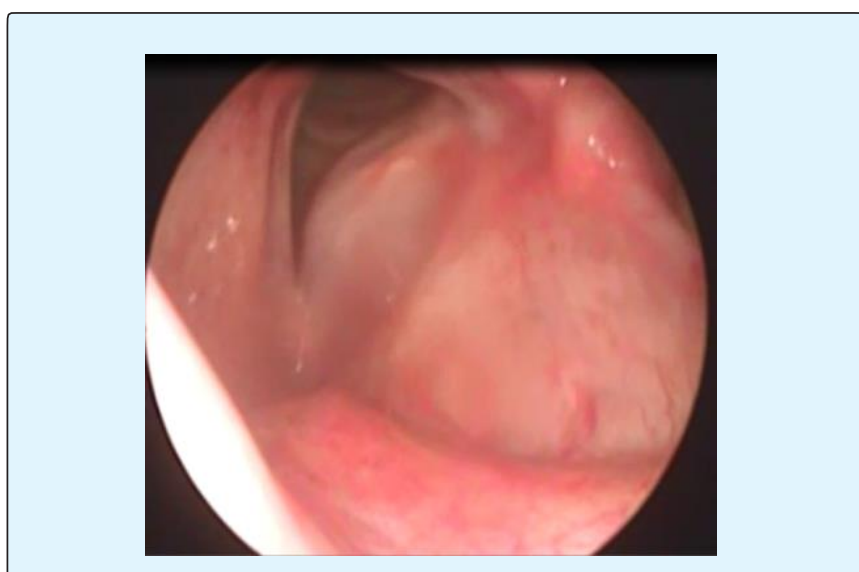

Figure 1: Laryngeal lesion occupying the left true vocal cord.

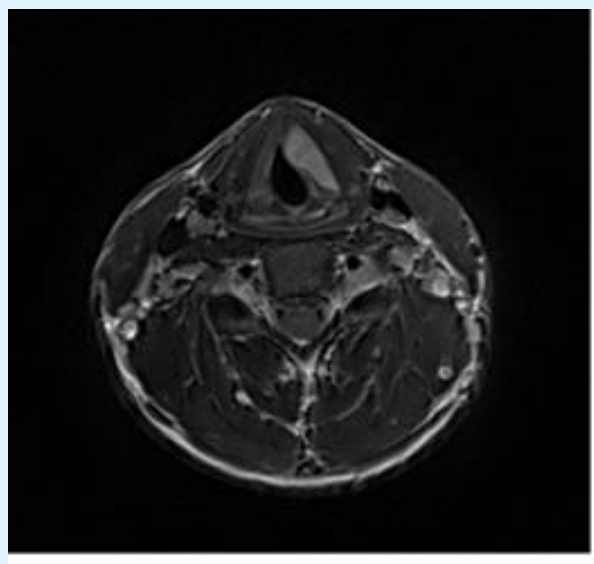

$2 \mathrm{~A}$

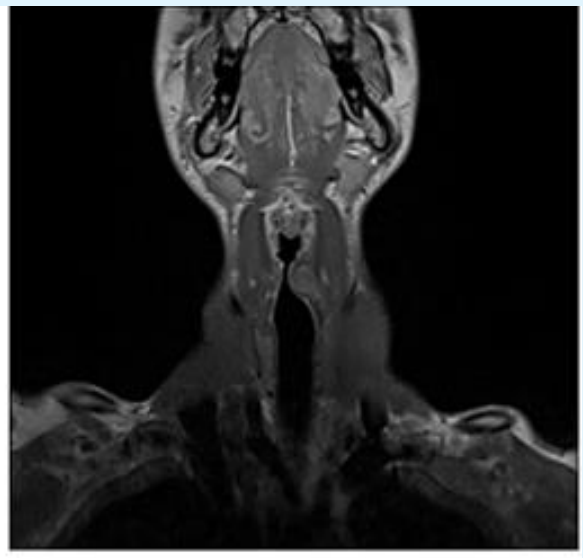

2B

Figure 2a \& $\mathbf{2 b}$ : Lesion of $2.4 \mathrm{~cm}$ anteroposterior diameter, $1.27 \mathrm{~cm}$ laterolateral diameter, occupying the left true vocal cord and extending in the paraglottide space (2A transverse plan, 2B coronal plan, high signal sequence).

Based on these findings and the patient's medical history, a diagnosis of benign neck and laryngeal tumor was made. Clinical and imaging features indicated a laryngeal neurofibroma. A direct laryngoscopy was performed under general anaesthesia and after ensuring the airway via orotracheal intubation, the lesion was completely resected with $\mathrm{CO}_{2}$ laser under microscopic control (Figure 3). The mass was dissected from the vocal cord without any significant blood loss or normal tissue damage.
The patient received intravenous antibiotics and corticosteroids in order to avoid any possible postoperative laryngeal edema or inflammation, and was released from the hospital the next day with complete relief of the symptoms. We suggested to avoid smoking postoperatively and not to use his voice for 2 days (Figure $4)$. The histopathologic exam revealed a tumor of the peripheral nerves, 2,2 cm of diameter with morphological and immunohistochemical characteristics (S100 protein, CD34 and EMA staining) of neurofibroma without any 


\section{Otolaryngology Open Access Journal}

sign of malignancy. The patient was under periodic follow-up the next year. The endoscopic evaluation of the larynx showed no relapse of the mass, and normal mobility of both true vocal cords (Figure 5).

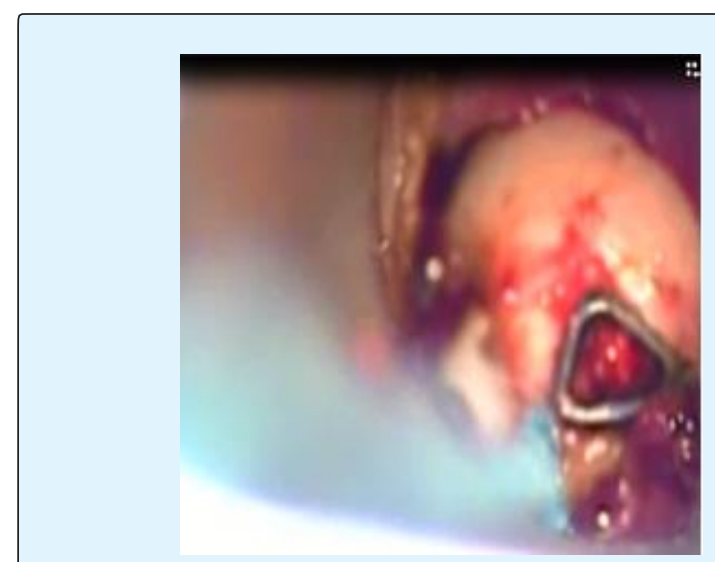

Figure 3: Intraoperative- resection of the laryngeal fibroma.

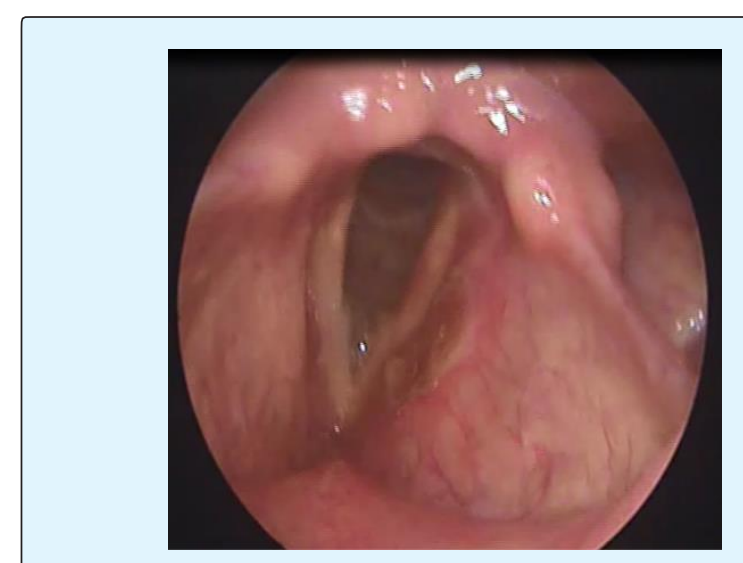

Figure 4: Postoperative image-the next day.

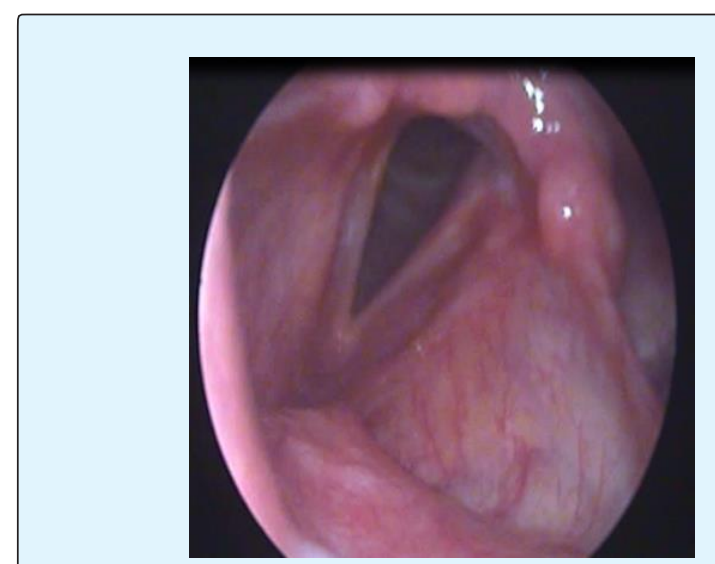

Figure 5: Postoperative image-a month after.

\section{Discussion}

Neurofibromas are peripheral nerve sheath tumours derived from peripheral nerves and they are associated with NF-1 and NF-2 or as spontaneous solitary lesions [3]. Neurofibromatosis-1 was first described by von Recklinghausen [4]. The disease is an autosomal dominant disorder, although $30-50 \%$ of cases are associated with spontaneous mutations. Neurofibromatosis-1 typically presents in childhood. Diagnostic criteria of NF-1 are defined as presentation with 2 or more of the following: café-au-lait spots, neurofibromas, axillary/groin freckling, optic glioma, more than 2 Lisch nodules, sphenoid or long bone dysplasia, or first-degree relative with NF-1. Prevalence of NF-1 is cited in the literature as 1 in 2500 to 3000 patients. Neurofibromatosis- 2 is an even rarer syndrome occurring in 1:40,000 live births with presentation typically in the third decade of life. Like NF-1, it is associated with neurofibromas and transmitted in an autosomal dominant fashion. Bilateral acoustic neuromas are pathognomonic for NF-2, as well as the extra-cranial neurofibromas [5-7].

Laryngeal neurofibromas are rare tumours in population with NF-1. Chevalier Jackson was the first to describe LNF while Colledge in 1930 was the first to describe LNF associated with neurofibromatosis $[8,9]$. Most of these lesions have been reported in the pediatric population and in association with NF-1 [10]. The aryepiglottic fold is the anatomic site most commonly involved [11] (34\%), followed by the arytenoid (18\%) and the post-cricoid/hypopharynx (14\%). Laryngeal neurofibromas arises from the superior laryngeal branch of the glossopharyngeal nerve. No malignant changes occur among isolated neurofibromas. The progression from a solitary neurofibroma to multiple neurofibromatosis and then transformation into malignancy is theoretically possible but exceedingly rare [9].

Although the incidence of LNF in NF-1 patients is low, any patient with NF-1 who presents with airway symptoms, voice changes or swallowing difficulty warrants further evaluation. The most common symptoms that patients with laryngeal neurofibromas present, are dysphagia, hoarseness of the voice, dysphonia, dyspnea and stridor. There is no indication for medication trials and complete surgical excision is the primary therapy [10]. Bagwell in 1990 was the first to report an endoscopic removal of a $\mathrm{LNF}$ with a $\mathrm{CO}_{2}$ laser [11]. The use of endoscopic $\mathrm{CO}_{2}$ laser consists of a minimally invasive technique, safer that any open 


\section{Otolaryngology Open Access Journal}

technique, with less complication and no need for tracheostomy, a condition extremely amputative for a patient.

\section{References}

1. Ji Y, Xu B, Wang X, Liu W, Chen S (2011) Surgical treatment of giant plexiform neurofibroma associated with pectus excavatum. J Cardiothorac Surg 6: 119.

2. Liu J, Wong CF, Lim F, Kanagalingam J (2013) Glottic neurofibroma in an elderly patient: a case report. J Voice 27(5): 644-646.

3. White AK, Smith RJ, Bigler CR, Brooke WF, Schauer PR (1986) Head and neck manifestations of neurofibromatosis. Laryngoscope 96(7): 732-737.

4. von Recklinghausen FD (1882) Uber die multiplen Fibromeder Haut und ihre Beziehung zuden multiplen Neuromen. Berlin, Germany: A Hirschwald.

5. Chang Lo M (1977) Laryngeal involvement in Von Recklinghausen's disease: a case report and review of the literature. Laryngoscope 87(3): 435-442.
6. Rahbar R, Litrovnik BG, Vargas SO, Robson CD, Nuss RC, et al. (2004) The biology and management of laryngeal neurofibroma. Arch Otolaryngol Head Neck Surg 130(12): 1400-1406.

7. Fukuda I, Ogasawara H, Kumoi T, Sugihara K, Wada $\mathrm{H}$ (1987) Subglottic neurofibroma in a child. Int J Pediatr Otorhinolaryngol 14(3): 161-170.

8. Figi FA, Stark DB (1953) Neurofibroma of the larynx; presentation of five cases. Laryngoscope 63(7): 652659.

9. Chen YW, Fang TJ, Li HY (2004) A solitary laryngeal neurofibroma in a pediatric patient. Chang Gung Med J 27(12): 930-933.

10. Washington EN, Placket TP, Gagliano RA, Kavolius J, Person DA (2010) Diffuse plexiform neurofibroma of the back: report of a case. Hawaii Med J 69(8): 191193.

11. Bagwell CE (1990) CO2 laser excision of pediatric airway lesions. J Pediatr Surg 25(11): 1152-1156. 